\title{
Measuring semantic similarity of words using concept networks
}

\author{
Gábor Recski \\ Research Institute for Linguistics \\ Hungarian Academy of Sciences \\ H-1068 Budapest, Benczúr u. 33 \\ recski@mokk. bme. hu
}

\author{
Katalin Pajkossy \\ Department of Algebra \\ Budapest U of Technology and Economics \\ H-1111 Budapest, Egry J. u. 1 \\ pajkossy@mokk. bme.hu
}

\author{
Eszter Iklódi \\ Dept of Automation and Applied Informatics \\ Budapest $U$ of Technology and Economics \\ H-1117 Budapest, Magyar tudósok krt. 2 \\ eszter.iklodi@gmail.com
}

\author{
András Kornai \\ Institute for Computer Science \\ Hungarian Academy of Sciences \\ H-1111 Budapest, Kende u. 13-17 \\ andrasekornai.com
}

\begin{abstract}
We present a state-of-the-art algorithm for measuring the semantic similarity of word pairs using novel combinations of word embeddings, WordNet, and the concept dictionary 4 lang. We evaluate our system on the SimLex-999 benchmark data. Our top score of 0.76 is higher than any published system that we are aware of, well beyond the average inter-annotator agreement of 0.67 , and close to the 0.78 average correlation between a human rater and the average of all other ratings, suggesting that our system has achieved nearhuman performance on this benchmark.
\end{abstract}

\section{Introduction}

We present a hybrid system for measuring the semantic similarity of word pairs. The system relies both on standard word embeddings, the WordNet database, and features derived from the 4 lang concept dictionary, a set of concept graphs built from entries in monolingual dictionaries of English. 4 lang-based features improve the performance of systems using only word embeddings and/or WordNet, our top configurations achieve state-of-the-art results on the SimLex-999 data, which has recently become a popular benchmark of word similarity metrics.

In Section 1 we summarize earlier work on measuring word similarity and review the latest results achieved on the SimLex-999 data. Section 2 describes our experimental setup, Sections 2.1 and 2.2 documents the features obtained using word embeddings and WordNet. In Section 3 we briefly introduce the 4 lang resources and the formalism it uses for encoding the meaning of words as directed graphs of concepts, then document our efforts to develop novel 4 langbased similarity features. Besides improving the performance of existing systems for measuring word similarity, the goal of the present project is to examine the potential of $4 \mathrm{l}$ ang representations in representing non-trivial lexical relationships that are beyond the scope of word embeddings and standard linguistic ontologies.

Section 4 presents our results and provides rough error analysis. Section 5 offers some conclusions and plans for future work. All software presented in this paper is available for download under an MIT license at http://github.com/recski/wordsim.

\section{Background}

Measuring the semantic similarity of words is a fundamental task in various natural language processing applications. The ability to judge the similarity in meaning of any two linguistic structures reflects on the quality of the representations used. Vector representations (word embeddings) are commonly used as the component encoding (lexical) semantics in virtually all NLP applications. The similarity of word vectors is by far the most common source of information for semantic similarity in state-of-the-art systems, e.g. nearly all top-scoring systems at the 2015 SemEval Task on measuring semantic similarity (Agirre et al., 2015) rely on word embeddings to score sentence pairs (see e.g. (Sultan et al., 2015; Han et al., 
2015)).

Hill et al. (2015) proposed the SimLex-999 dataset as a benchmark for word similarity, arguing that pre-existing gold standards measure association, not similarity, of word pairs; e.g. the words cup and coffee receive a high score by annotators in the widely used wordsim353 data (Finkelstein et al., 2002). SimLex has since been used to evaluate various algorithms for measuring word similarity. Hill et al. (2015) reports a Spearman correlation of 0.414 achieved by an embedding trained on Wikipedia using word2vec (Mikolov et al., 2013). Schwartz et al. (2015) achieves a score of 0.56 using a combination of a standard word2vec-based embedding and the SP model, which encodes the cooccurrence of words in symmetric patterns such as $X$ and $Y$ or $X$ as well as $Y$.

Banjade et al. (2015) combined multiple word embeddings with the word similarity algorithm of (Han et al., 2015) used in a top-scoring SemEval system, and simple features derived from WordNet (Miller, 1995) indicating whether word pairs are synonymous or antonymous. Their top system achieved a correlation of 0.64 on SimLex. The highest score we are aware of is achieved using the Paragram embedding (Wieting et al., 2015), a set of vectors obtained by training preexisting embeddings on word pairs from the Paraphrase Database (Ganitkevitch et al., 2013). The top correlation of 0.69 is measured when using 300-dimension embedding created from the same GloVe-vectors that have been introduced in this section (trained on 840 billion tokens). Hyperparameters of this database have been tuned for maximum performance on SimLex, another version tuned for the WS-353 dataset achieves a correlation of 0.667 .

\section{Setup}

Our system is trained on a variety of real-valued and binary features generated using word embeddings, WordNet, and 4 lang definition graphs. Each class of features will be presented in detail below. We perform support vector regression (with RBF kernel) over all features using the numpy library, the model is trained on 900 pairs of the SimLex data and used to obtain scores for the remaining 99 pairs. We compute the Spearman correlation of the output with SimLex scores. We evaluate each of our models using tenfold crossvalidation and by averaging the ten correlation figures. The changes in performance caused by previously used feature classes are described next, the performance of all major configurations are summarized in Section 4.

\subsection{Word embeddings}

Features in the first group are based on word vector similarity. For each word pair the cosine similarity of the corresponding two vectors is calculated for all embeddings used. Three sets of word vectors in our experiments were built using the neural models compared by Hill et al. (2015): the SENNA ${ }^{1}$ (Collobert and Weston, 2008), and Huang $^{2}$ (Huang et al., 2012) embeddings contain 50-dimension vectors and were downloaded from the authors' webpages. The word2vec (Mikolov et al., 2013) vectors are of 300 dimensions and were trained on the Google News dataset ${ }^{3}$.

We extend this set of models with GloVe vectors ${ }^{4}$ (Pennington et al., 2014), trained on 840 billion tokens of Common Crawl data ${ }^{5}$, and the two word embeddings mentioned in Section 1 that have recently been evaluated on the SimLex dataset: the 500-dimension SP model ${ }^{6}$ (Schwartz et al., 2015) (see Section 1) and the 300-dimension Paragram vectors ${ }^{7}$ (Wieting et al., 2015). The model trained on 6 features corresponding to the 6 embeddings mentioned achieves a Spearman correlation of 0.72 , the performance of individual embeddings is listed in Table 1.

\subsection{Wordnet}

Another group of features are derived using WordNet (Miller, 1995). WordNet-based metrics proved to be useful in the Semeval-system of Han et al. (2013), who used these metrics for calculating a boost of word similarity scores. The top system of Banjade et al. (2015) also includes a subset of these features. We chose to use four of these metrics as binary features in our system;

\footnotetext{
${ }^{1}$ http: //ronan. collobert.com/senna/

${ }^{2}$ http: / / www. socher.org

${ }^{3}$ https: / / code.google.com/archive/p/ word2vec/

${ }^{4}$ http://nlp.stanford.edu/projects/ glove/

${ }^{5}$ https: / / commoncrawl.org/

${ }^{6}$ http://www.cs.huji.ac.il/ roys02/ papers/sp_embeddings/sp_embeddings.html

${ }^{7}$ http: //ttic.uchicago.edu/ wieting/
} 


\begin{tabular}{lr}
\hline System & Spearman's $\rho$ \\
\hline Huang & 0.14 \\
SENNA & 0.27 \\
GloVe & 0.40 \\
Word2Vec & 0.44 \\
SP & 0.50 \\
Paragram & 0.68 \\
\hline 6 embeddings & $\mathbf{0 . 7 2}$ \\
\hline
\end{tabular}

Table 1: Performance of word embeddings on SimLex

these indicate whether one word is a direct or twolink hypernym of the other, whether the two are derivationally related, and whether one word appears frequently in the glosses of the other (and its direct hypernym and its direct hyponyms). Each of these features improved our system independently, adding all of them brought the system's performance to 0.73 . A model trained on the 4 WordNet-based features alone achieves a correlation of 0.33 .

\section{4lang}

The 4 lang theory of semantics was introduced and motivated in Kornai (2010) and Kornai (2012). The name refers to the initial concept dictionary, which had bindings in four languages, representative samples of the major language families spoken in Europe; Germanic (English), Slavic (Polish), Romance (Latin), and Finno-Ugric (Hungarian). Today, bindings exist in over 40 languages (Ács et al., 2013). We only present a bird's-eye view here, and refer the reader to the book-length presentation (Kornai, in preparation) for details. In brief, 4 lang is an algebraic (symbolic) system that puts the emphasis on lexical definitions at the word and sub-word level, and on valency (slot-filling) on the phrase and sentence level. Paragraphs and yet higher (discourse) units are not well worked out, but these play no role in any of the approaches to analogy and similarity that we are aware of.

Historically, 4 lang falls in the $\mathrm{AI} / \mathrm{KR}$ tradition, following on the work of Quillian (1969), Schank (1975), and more recently Banarescu et al. (2013). Linguistically, it is closest to Wierzbicka (1972), Goddard (2002) and to modern theories of case grammar and linking theory (see Butt (2006) for a summary). Computationally, 4 lang is in the finite state tradition (Koskenniemi, 1983), except it relies on an extension of finite state automata (FSA) introduced by Eilenberg (1974) to machines.

In addition to the usual state machine (where letters of the alphabet correspond to directed edges running between the states), an Eilenberg machine will also have a base set $X$, with each letter of the alphabet corresponding to a binary relation over $X$. As the machine consumes letters one by one, the corresponding relations are composed. How this mechanism can be used to account for slotfilling in a variable-free setting is described in Kornai (2010).

Central to the goals of the current paper is the structure of $X$. As a first approximation, $X$ can be thought of as a hypergraph, where each hypernode is a lexeme (for a total of about $10^{5}$ such hypernodes), and hyperedges run from (hyper)node a to $b$ if $b$ appears in the definition of $a$. Since the definition of fox includes the word clever, we have a link from fox to clever, but not conversely, since the definition of clever does not refer to fox. Edges are of three types: 0, corresponding both to attribution and IS_A relations; 1, corresponding to grammatical subjects; and 2, corresponding to grammatical objects. Indirect objects are handled by the decomposition methods pioneered in generative semantics, without recourse to a ' 3 ' link type (Kornai, 2012).

Each lexeme is a small Eilenberg machine, with only a few states in its FSA, so the state space $X$ of the entire lexicon is best viewed as a large graph with about $10^{6}$ states (assuming 10 states per hypernode). This base set is shared across the individual machines and functions analogously to the blackboard long familiar from AI (Nii, 1986). The primary purpose of the machine apparatus is to formalize the classical distributed model of semantic interpretation, spreading activation (Collins and Loftus, 1975; Nemeskey et al., 2013), by a series of changes in the hypernode activation levels, described by the relations on X. Manual grammar writing in this style can lead to very high precision high recall grammars (Karlsson et al., 1995; Tapanainen and Järvinen, 1997), but for now we rely on the Stanford Parser (Chen and Manning, 2014) to produce the dependency structures that we process into simplified 
$4 \mathrm{l}$ ang representations (ordinary edge-colored directed graphs rather than hypergraphs) we call definition graphs and describe briefly in Section 3.1.

We derive several similarity features from pairs of definition graphs built using the 4 lang library $^{8}$. Words that are not part of the manually built 4 lang dictionary ${ }^{9}$ are defined by graphs built from entries in monolingual dictionaries of English using the Stanford Dependency Parser and a small hand-written mapping from dependency relations to 4 lang connections (see Recski (2016) for details). The set of all words used in definitions of the Longman Dictionary of Contemporary English (Bullon, 2003), also known as the Longman Defining Vocabulary (LDV), is included in the ca. 3000 words that are defined manually in the 4 lang dictionary. Recski and Ács (2015) used a word similarity metric based on 4 lang graphs in their best STS submission, their findings served as our starting point when defining features over pairs of 4 lang graphs.

\subsection{The formalism}

For the purposes of word similarity calculations we find it expedient to abstract away from some of the hypergraph/machine aspects of 4 lang discussed above and represent the meaning of both words and utterances as directed graphs, similarly to the Abstract Meaning Representations (AMRs) of Banarescu et al. (2013). Nodes correspond to language-independent concepts, edges may have one of three labels $(0,1,2)$. 0 -edges represent attribution $(\operatorname{dog} \stackrel{0}{\rightarrow}$ friendly), the IS_A relation (hypernymy) (dog $\stackrel{0}{\rightarrow}$ animal), and unary predication (dog $\stackrel{0}{\rightarrow}$ bark). Since concepts do not have grammatical categories, phrases like $w a$ ter freezes and frozen water would both be represented as water $\stackrel{0}{\rightarrow}$ freeze. 1 - and 2-edges connect binary predicates to their arguments, e.g. cat $\stackrel{1}{\leftarrow}$ catch $\stackrel{2}{\rightarrow}$ mouse). The meaning of each 4 lang concept is represented as a 4 lang graph over other concepts, e.g. the concept bird is defined by the graph in Figure 1.

\subsection{Graph-based features}

We experimented with various features over pairs of 4 lang graphs as a source of word

\footnotetext{
${ }^{8}$ http://www.github.com/kornai/4lang

${ }^{9}$ http://hlt.bme.hu/en/resources/4lang_ dict
}

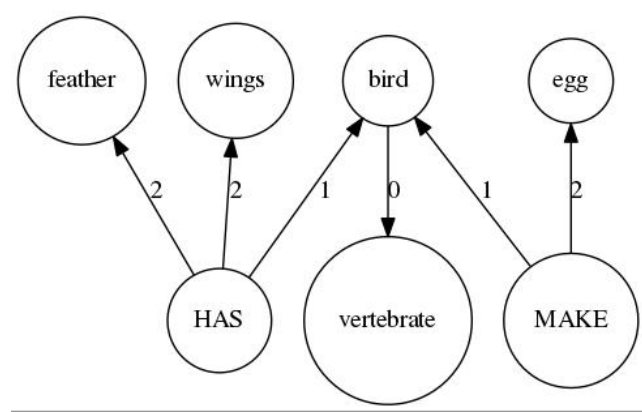

Figure 1: 4lang definition of bird.

similarity. The simple metric defined by Recski and Ács (2015) is based on the intuition that similar concepts will overlap in the elementary configurations they take part in: they might share a 0 -neighbor, e.g. train $\stackrel{0}{\rightarrow}$ vehicle $\stackrel{0}{\leftarrow}$ car, or they might be on the same path of 1- and 2-edges, e.g. park $\stackrel{1}{\leftarrow}$ IN $\stackrel{2}{\rightarrow}$ town and street $\stackrel{1}{\leftarrow}$ IN $\stackrel{2}{\rightarrow}$ town. The metric used by Recski and Ács (2015) defines the sets of predicates of each concept based on this intuition: given the example definition of bird in Figure 1, predicates of the concept bird ( $P($ bird $)$ ) are \{vertebrate; (HAS, feather); (HAS, wing); (MAKE, egg)\}. Predicates are also inherited via paths of 0-edges, e.g. (HAS, wing) will be a predicate of all concepts for which $\stackrel{0}{\rightarrow}$ bird holds.

Our first feature extracted for each word pair is the Jaccard similarity of the sets of predicates of each concept, i.e.

$$
S\left(w_{1}, w_{2}\right)=\frac{\left|P\left(w_{1}\right) \cap P\left(w_{2}\right)\right|}{\left|P\left(w_{1}\right) \cup P\left(w_{2}\right)\right|}
$$

A second similar feature takes into account all nodes accessible from each concept in its definition graph. Recski and Ács (2015) observe that this allows us to capture minor similarities between concepts, e.g. the definitions of casualty and army do not share predicates but do have a common node war (see Figure 2).

Based on boosting factors in the original metric we also generated three binary features. The links_contain feature is true iff either concept is contained in a predicate of the other, nodes_contain holds iff either concept is included in the other's definition graph, and 0_connected is true if the two nodes are connected by a path of 0 -edges in either definition 


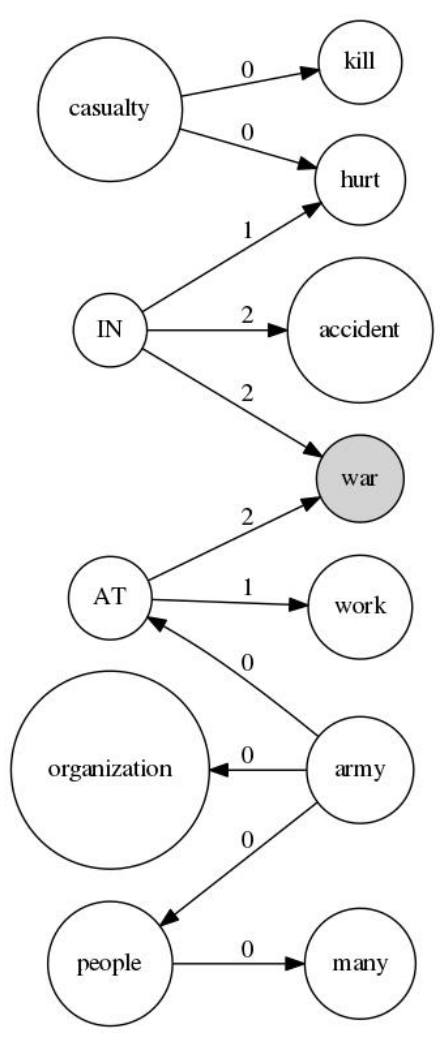

Figure 2: Overlap in the definitions of casualty (built from LDOCE) and army (defined in 4lang)

\begin{tabular}{ll}
\hline feature & definition \\
\hline links_jaccard & $J\left(P\left(w_{1}\right), P\left(w_{2}\right)\right)$ \\
nodes_jaccard & $J\left(N\left(w_{1}\right), N\left(w_{2}\right)\right)$ \\
\hline links_contain & iff $w_{1} \in P\left(w_{2}\right)$ or $w_{2} \in P\left(w_{1}\right)$ \\
nodes_contain & iff $w_{1} \in N\left(w_{2}\right)$ or $w_{2} \in N\left(w_{1}\right)$ \\
\hline 0_connected & iff $w_{1}$ and $w_{2}$ are on a path of 0 -edges \\
\hline
\end{tabular}

Table 2: 4 lang word similarity features

graph. All features are listed in Table 2.

The dict_to_4lang module used to build graphs from dictionary definitions allowed us to perform expansion on each graph, which involves adjoining the definition graphs of all words to the initial graph; an example is show in Figure 3.

Using only these features in initial experiments resulted in many "false positives": pairs of antonyms in SimLex were often assigned high similarity scores because this feature set is not sensitive to the 4 lang nodes LACK, representing negation (dumb $\stackrel{0}{\rightarrow}$ intelligent $\stackrel{0}{\rightarrow}$ LACK), and $B E F O R E$, indicating that something was only true in the past (forget $\stackrel{0}{\rightarrow}$ know $\stackrel{0}{\rightarrow}$ before),

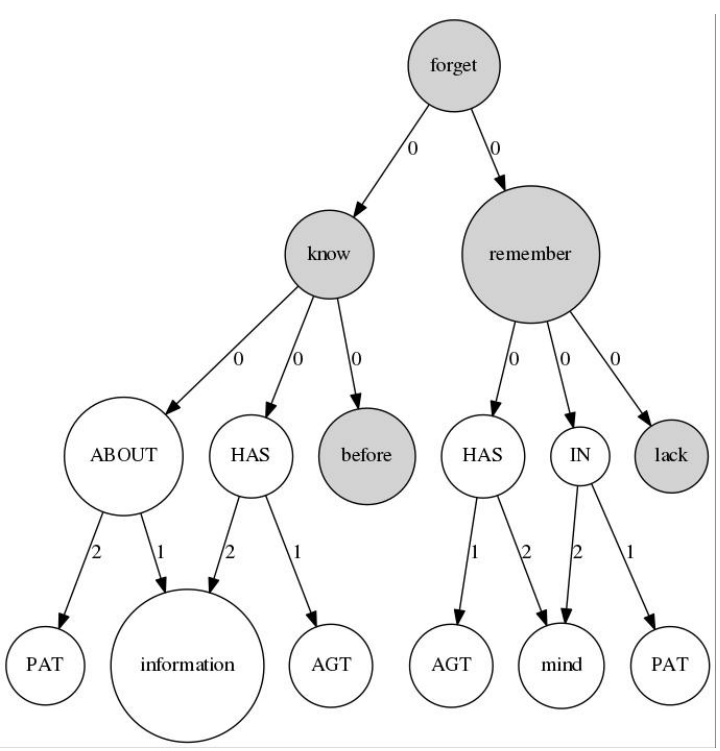

Figure 3: Expanded 4 lang definition of forget. Nodes of the unexpanded graph are shown in gray.

We attempt to model the effect of these nodes in two ways. First, we implement the is_antonym feature, a binary set to true if one word is within the scope (i.e. 0-connected to) an instance of either lack or before in the other word's graph. Next, we transform the input graphs of remaining features so that all nodes within the scope of lack or before are prefixed by $\mathrm{lack}_{\text {- }}$ and are not considered identical with their non-negated counterparts when computing each of the features in Table 2. An example of such a transformation is shown in Figure 4.

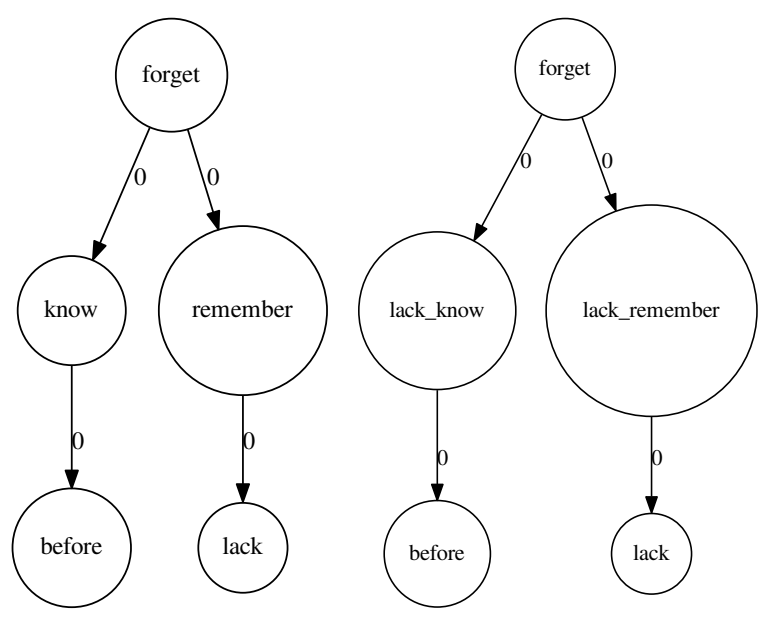

Figure 4: 4 lang definition of forget and its modified version 
Early experiments show that a system trained on 4 l ang-based features only can achieve a Pearson correlation in the range of $0.32-0.34$ on the SimLex data, this was increased to 0.38 by the handling of LACK and BEFORE described above. This score is competitive with some word embeddings, but well below the $0.58-0.68$ range achieved by the state-of-the-art vector-based systems cited in Section 1 and reproduced in Section 2.1.

After testing 4 lang features' impact on purely vector-based configurations we came to the conclusion that the only 4 lang-based features that improve their performance significantly are 0 -connected and is_antonym. Adding these two features to the vector-based system brings correlation to 0.76 .

\section{Results}

Performance of our main configurations is presented in Table 3. The system relying on word embeddings achieves a Spearman correlation of 0.72 . WordNet and 4 lang features both improve the vector-based system, combining all three feature classes yields our top correlation of 0.76 , higher than any previously published results. Since the average correlation between a human rater and the average of all other raters is 0.78 , this figure suggests that our system has achieved near-human performance on this benchmark.

\begin{tabular}{lr}
\hline System & Spearman's $\rho$ \\
\hline embeddings & 0.72 \\
\hline embeddings+wordnet & 0.73 \\
\hline embeddings+4lang & 0.75 \\
\hline embeddings+wordnet+4lang & $\mathbf{0 . 7 6}$ \\
\hline
\end{tabular}

Table 3: Performance of major configurations on SimLex

For the purposes of error analysis we sorted word pairs by the difference between gold similarity values from $\mathrm{S}$ imLex and the output of our topscoring model. The top of this list is clearly dominated by two error classes. The largest group consists of (near-)synonyms that have not been identified as related by our model, Table 4 shows the top 5 word pairs from this category. The second error group contains word pairs that have been falsely rewarded for being associated, but not similar by the definition used when creating the SimLex data. Table 5 shows the top 5 word pairs of this error class. This second error class is an indication of a well-known shortcoming of word similarity models: (Hill et al., 2015) observes that similarity of vectors in word embeddings tend to encode association (or relatedness) rather than the similarity of concepts.

\begin{tabular}{llrrr}
\hline word1 & word2 & output & gold & diff \\
\hline bubble & suds & 2.97 & 8.57 & 5.59 \\
dense & dumb & 1.71 & 7.27 & 5.56 \\
cop & sheriff & 3.50 & 9.05 & 5.55 \\
alcohol & gin & 3.43 & 8.65 & 5.22 \\
rationalize & think & 3.50 & 8.25 & 4.75 \\
\hline
\end{tabular}

Table 4: Top 5 "false negative" errors

\begin{tabular}{llrrr}
\hline word1 & word2 & output & gold & diff \\
\hline girl & maid & 7.72 & 2.93 & -4.79 \\
happiness & luck & 6.59 & 2.38 & -4.21 \\
crazy & sick & 7.49 & 3.57 & -3.92 \\
arm & leg & 6.74 & 2.88 & -3.86 \\
breakfast & supper & 8.01 & 4.40 & -3.61 \\
\hline
\end{tabular}

Table 5: Top 5 "false positive" errors

Since our main purpose was to experiment with 4 lang representations and identify its shortcomings, we examined 4 lang graphs of top erroneous word pairs. As expected, the value of the 0 -connected feature was -1 for each "false negative" pair, i.e. word pairs such as those in Table 4 were not on the same path of 0edges. In most cases this is due to the current lack of simple inferencing on 4 lang representations. For example, suds are defined in LDOCE as the mass of bubbles formed on the top of water with soap in it, yet the resulting 4 lang subgraph bubble $\stackrel{1}{\leftarrow}$ HAS $\stackrel{2}{\rightarrow}$ mass $\stackrel{0}{\leftarrow}$ suds will not trigger any mechanism that would derive suds $\stackrel{0}{\rightarrow}$ bubble. Inference will also be responsible for deriving all uses of polysemous words, the 4 lang representation of dense is therefore built from its first definition in LDOCE: made of or containing a lot of things or people that are very close together. A method of inference that will relate this definition with that of dumb is clearly out of reach. Better short-term results could be 
obtained by using all definitions in a dictionary to build 4 lang representations, for dense this would include its third definition: not able to understand things easily.

Other shortcomings of 4 lang representations are of a more technical nature, e.g. the lemmatizer used to map words of definitions to concepts failed to map alcoholic to alcohol in the definition of gin: a strong alcoholic drink made mainly from grain. Yet other errors could be addressed by rewarding the overlap between two representations, e.g. that the graphs for cop and sheriff both contain $\stackrel{0}{\rightarrow}$ officer.

\section{Conclusions, future work}

The purpose of experimenting with 4 lang-based features was to gain a better understanding of how 4 lang may implicitly encode semantic relations that are difficult to model with standard tools such as word embeddings or WordNet. We found that simple features describing the relation between two concepts in 4 lang improve vectorbased systems significantly. Since less explicit relationships may be encoded by more distant relationships in the network of 4 lang concepts, in the future we plan to examine portions of this network larger than the union of two (expanded) definition graphs. Errors made by 4 lang-based systems also indicate that a more sophisticated form of lexical inference on 4 lang graphs may be necessary to establish the more distant connections between pairs of concepts. In the near future we plan to experiment with features defined on larger 4 lang networks. We also plan to extend our system to include the task of measuring phrase similarity, which can also be pursued using supervised learning given new resources such as the Annotated-PPDB and ML-Paraphrase datasets introduced by (Wieting et al., 2015).

\section{References}

Judit Ács, Katalin Pajkossy, and András Kornai. 2013. Building basic vocabulary across 40 languages. In Proceedings of the Sixth Workshop on Building and Using Comparable Corpora, pages 52-58, Sofia, Bulgaria. ACL.

Eneko Agirre, Carmen Banea, Claire Cardie, Daniel Cer, Mona Diab, Aitor Gonzalez-Agirre, Weiwei Guo, Iñigo Lopez-Gazpio, Montse Maritxalar, Rada Mihalcea, German Rigau, Larraitz Uria, and Janyce
Wiebe. 2015. SemEval-2015 Task 2: Semantic Textual Similarity, English, Spanish and Pilot on Interpretability. In Proceedings of the 9th International Workshop on Semantic Evaluation (SemEval 2015), Denver, CO, U.S.A.

Laura Banarescu, Claire Bonial, Shu Cai, Madalina Georgescu, Kira Griffitt, Ulf Hermjakob, Kevin Knight, Philipp Koehn, Martha Palmer, and Nathan Schneider. 2013. Abstract meaning representation for sembanking. In Proceedings of the 7th Linguistic Annotation Workshop and Interoperability with Discourse, pages 178-186, Sofia, Bulgaria. Association for Computational Linguistics.

Rajendra Banjade, Nabin Maharjan, Nobal B. Niraula, Vasile Rus, and Dipesh Gautam. 2015. Lemon and tea are not similar: Measuring word-to-word similarity by combining different methods. In Alexander Gelbukh, editor, Proc. CICLING15, pages 335-346. Springer.

Stephen Bullon. 2003. Longman Dictionary of Contemporary English 4. Longman.

Miriam Butt. 2006. Theories of Case. Cambridge University Press.

Danqi Chen and Christopher D Manning. 2014. A fast and accurate dependency parser using neural networks. In EMNLP, pages 740-750.

A.M. Collins and E.F. Loftus. 1975. A spreadingactivation theory of semantic processing. Psychological Review, 82:407-428.

Ronan Collobert and Jason Weston. 2008. A unified architecture for natural language processing: Deep neural networks with multitask learning. In Proceedings of the 25th International Conference on Machine Learning, ICML '08, pages 160-167, New York, NY, USA. ACM.

Samuel Eilenberg. 1974. Automata, Languages, and Machines, volume A. Academic Press.

Lev Finkelstein, Evgeniy Gabrilovich, Yossi Matias, Ehud Rivlin, Zach Solan, Gadi Wolfman, , and Eytan Ruppin. 2002. Placing search in context: The concept revisited. ACM Transactions on Information Systems, 20(1):116-131, January.

Juri Ganitkevitch, Benjamin Van Durme, and Chris Callison-Burch. 2013. Ppdb: The paraphrase database. In HLT-NAACL, pages 758-764.

Cliff Goddard. 2002. The search for the shared semantic core of all languages. In Cliff Goddard and Anna Wierzbicka, editors, Meaning and Universal Grammar - Theory and Empirical Findings, volume 1, pages 5-40. Benjamins.

Lushan Han, Abhay Kashyap, Tim Finin, James Mayfield, and Jonathan Weese. 2013. 
UMBC_EBIQUITY-CORE: Semantic textual similarity systems. In Proceedings of the 2nd Joint Conference on Lexical and Computational Semantics, pages 44-52.

Lushan Han, Justin Martineau, Doreen Cheng, and Christopher Thomas. 2015. Samsung: Align-andDifferentiate Approach to Semantic Textual Similarity. In Proceedings of the 9th International Workshop on Semantic Evaluation (SemEval 2015), pages 172-177, Denver, Colorado. Association for Computational Linguistics.

Felix Hill, Roi Reichart, and Anna Korhonen. 2015. Simlex-999: Evaluating semantic models with (genuine) similarity estimation. Computational Linguistics.

Eric H. Huang, Richard Socher, Christopher D. Manning, and Andrew Y. Ng. 2012. Improving word representations via global context and multiple word prototypes. In Proceedings of the 50th Annual Meeting of the Association for Computational Linguistics: Long Papers - Volume 1, ACL'12, pages 873882, Stroudsburg, PA, USA. Association for Computational Linguistics.

Fred Karlsson, Atro Voutilainen, Juha Heikkila, and Arto Anttila. 1995. Contraint Grammar, A Language-Independent System for Parsing Unrestricted Text. Mouton de Gruyter, Berlin, New-York.

András Kornai. 2010. The algebra of lexical semantics. In Christian Ebert, Gerhard Jäger, and Jens Michaelis, editors, Proceedings of the 11th Mathematics of Language Workshop, LNAI 6149, pages 174-199. Springer.

András Kornai. 2012. Eliminating ditransitives. In $\mathrm{Ph}$. de Groote and M-J Nederhof, editors, Revised and Selected Papers from the 15th and 16th Formal Grammar Conferences, LNCS 7395, pages 243261. Springer.

András Kornai. in preparation. Semantics. http://kornai.com/Drafts/sem.pdf.

Kimmo Koskenniemi. 1983. Two-Level Morphology: A General Computational Model for WordForm Recognition and Production. $\mathrm{PhD}$ thesis, University of Helsinki.

Tomas Mikolov, Kai Chen, Greg Corrado, and Jeffrey Dean. 2013. Efficient estimation of word representations in vector space. In Y. Bengio and Y. LeCun, editors, Proceedings of the ICLR 2013.

George A. Miller. 1995. Wordnet: a lexical database for English. Communications of the ACM, 38(11):39-41.

Dávid Nemeskey, Gábor Recski, Márton Makrai, Attila Zséder, and András Kornai. 2013. Spreading activation in language understanding. In Proc. CSIT 2013, pages 140-143, Yerevan, Armenia. Springer.
H. Penny Nii. 1986. Blackboard application systems, blackboard systems and a knowledge engineering perspective. AI Magazine, 7(3):82-110.

Jeffrey Pennington, Richard Socher, and Christopher Manning. 2014. Glove: Global vectors for word representation. In Conference on Empirical Methods in Natural Language Processing (EMNLP 2014).

M. Ross Quillian. 1969. The teachable language comprehender. Communications of the ACM, 12:459476.

Gábor Recski and Judit Ács. 2015. MathLingBudapest: Concept networks for semantic similarity. In Proceedings of the 9th International Workshop on Semantic Evaluation (SemEval 2015), pages 543547, Denver, Colorado. Association for Computational Linguistics.

Gábor Recski. 2016. Building concept graphs from monolingual dictionary entries. In Nicoletta Calzolari, Khalid Choukri, Thierry Declerck, Marko Grobelnik, Bente Maegaard, Joseph Mariani, Asuncion Moreno, Jan Odijk, and Stelios Piperidis, editors, Proceedings of the Tenth International Conference on Language Resources and Evaluation (LREC 2016), Portorož, Slovenia. European Language Resources Association (ELRA).

Roger C. Schank. 1975. Conceptual Information Processing. North-Holland.

Roy Schwartz, Roi Reichart, and Ari Rappoport. 2015. Symmetric pattern based word embeddings for improved word similarity prediction. CoNLL 2015, page 258 .

Md Arafat Sultan, Steven Bethard, and Tamara Sumner. 2015. DLS@CU: Sentence similarity from word alignment and semantic vector composition. In Proceedings of the 9th International Workshop on Semantic Evaluation (SemEval 2015), pages 148153, Denver, Colorado. Association for Computational Linguistics.

Pasi Tapanainen and Timo Järvinen. 1997. A nonprojective dependency parser. In Proceedings of the 5th Conference on Applied Natural Language Processing, pages 64-71.

Anna Wierzbicka. 1972. Semantic Primitives. Athenäum, Frankfurt.

John Wieting, Mohit Bansal, Kevin Gimpel, Karen Livescu, and Dan Roth. 2015. From paraphrase database to compositional paraphrase model and back. TACL, 3:345-358. 